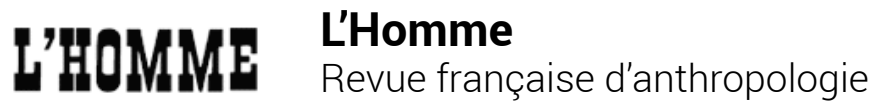

195-196 | 2010

Auto-biographie, Ethno-biographie

\section{Une valse du démon et de l'histoire}

A Walz with the Devil and History

\section{Sylvie Anne Goldberg}

\section{(2) OpenEdition}

\section{Journals}

Édition électronique

URL : http://journals.openedition.org/lhomme/22444

DOI : 10.4000/lhomme.22444

ISSN : 1953-8103

Éditeur

Éditions de l'EHESS

\section{Édition imprimée}

Date de publication : 10 novembre 2010

Pagination : 51-81

ISSN : 0439-4216

Référence électronique

Sylvie Anne Goldberg, "Une valse du démon et de l'histoire », L'Homme [En ligne], 195-196 | 2010, mis en ligne le 04 novembre 2012, consulté le 20 avril 2019. URL : http://journals.openedition.org/ Ihomme/22444 ; DOI : 10.4000/lhomme.22444 


\title{
Une valse du démon et de l'histoire
}

\author{
Sylvie Anne Goldberg
}

LORSQUE j’ai rencontré le Dybbouk, je le connaissais déjà, probablement depuis toujours. L'envahisseur m'était infiniment familier. L'âme défunte, errante à la recherche d'un corps où s'engouffrer, était tapie dans les évocations de l'univers d'ombres souvent ranimées par mes parents. Il prenait parfois la parole, parlait à leur place, se nichait, menaçant, derrière les rideaux et sous les meubles, prêt à bondir hors de la cave et à s'approprier le corps du moindre passant. Mais de celui qui avait déplacé la scène vers le théâtre, je ne savais rien. Comme pour nombre de ceux de ma génération, l'auteur s'était éclipsé derrière la pièce. Jouée régulièrement depuis 1918 par des générations d'acteurs, professionnels et amateurs, cette histoire étrange d'une banale histoire d'amour qui a mal tourné exhume du musée du folklore juif un univers hallucinant. Ce n'est pourtant qu'une histoire d'au-delà, de possédés comme il y en a tant. Mais elle laisse une empreinte surprenante sur ceux qui la voient, en s'accolant à leur être, comme un véritable dybbouk qui joue de toute la gamme des ressorts de l'imaginaire.

Une autre rencontre avec An-Sky s'est produite à la lecture de feuillets de papier photocopiés. Il s'agissait d'un questionnaire élaboré pour une enquête ethnographique qu'il avait lancée et menée dans la zone de résidence à partir de l'été 1912, jusqu'à ce que la guerre l'interrompe. Du berceau à la tombe, toutes les pratiques et croyances des juifs devaient être ainsi répertoriées, passées au tamis d'une grille de questions. Comment fait-on? Que dit-on? Que croit-on? Que raconte-t-on? À l'aube du XXe siècle, en ces années 1910-1912, l'espace où s'était déployée la tradition ancestrale s'amenuisait, semblait irrémédiablement s'évanouir. 
Il paraissait s'éteindre doucement, épuisé par les poussées de migrations, d'assimilation, d'urbanisation, les révolutions, et, peut-être, tout naturellement d'étouffement et de vieillesse. En interrogeant des vieillards et des plus jeunes, il s'agissait alors de conserver la trace de ce qu'ils avaient entendu, ce qu'ils avaient vu, de ce qu'on leur avait transmis et de tout ce qui pouvait encore constituer une spécificité des comportements, pratiques et croyances des juifs. La lecture de ce questionnaire ouvrait une fenêtre sur un monde encore frémissant, et je plaçais son auteur parmi mes rêves d'improbable chercheuse : un jour, un jour, sûrement, j'en ferai quelque chose. Puis, toute curiosité aiguisée, j'ai commencé à lire ses écrits, à m'immiscer dans ses souvenirs. Et plus il prenait forme, plus sa personne m'envahissait, plus sa complexité se faisait jour. L'homme lui-même finit par disparaître, enseveli sous la multitude des vies qu'il avait menées. L'envie de faire mieux connaitre tout à la fois sa personne et ses œuvres a alors commencé à me hanter. En faire un paradigme, l'incarnation d'un monde disparu, par le truchement duquel le temps s'abolirait, l'espace d'un livre.

\section{La biographie que j'aurais pu écrire}

Comment le saisir? Il n'est pas qu'un faiseur de mots. Il n'est pas qu'un faiseur d'histoire. Il n'est pas qu'un ethnographe. Il n'est pas qu'un journaliste. Il n'est pas qu'un nouvelliste. Il n'est pas qu'un député de la Douma. Il n'est pas qu'un militant social-révolutionnaire. Il n'est pas qu'un bon vivant. Il n'est pas qu'un crève-la-faim. Il n'est pas qu'un nationaliste juif. Il n'est pas que le secrétaire d'un illustre révolutionnaire. Il n'est pas qu'un membre de la Croix-Rouge. Il n'est pas qu'un homme vaincu. Il est un homme qui a fait de l'espoir sa vie. Il n'est plus qu'un nom au-dessus de piles de liasses et de boîtes dans les archives. Il existe d'ailleurs encore, enfoui parmi des dizaines de récits de souvenirs, embusqué dans les mémoires du début du $\mathrm{XX}^{\mathrm{e}}$ siècle, rédigés en russe et en yiddish.

Il est pourtant quelqu'un dont on a oublié la personne ; quelqu'un dont on n'a pas su grand-chose; quelqu'un dont on ne sait plus rien. Son nom passe parfois sur l'écran des cinémas d'art et d'essai, il gît dans des recueils de littérature russe. Il est de toutes les anthologies et cours de yiddish. Il fait partie du panthéon des souvenirs kitsch. Il a été tonitruant, rabelaisien, il aimait écrire et chanter, manger et séduire, conquérir et convaincre. Il a souvent eu froid, il a âprement souffert de la faim, il a rarement eu un toit. Parfois il n'avait ni chaussure ni manteau, parfois pas même un lit. 
Comment rendre compte de sa vie? De son œuvre? De sa personne? Par quels aspects l'aborder? Par sa vie? Sa place posthume? Sa présence forte dans ses écrits, sa voix, résolument insaisissable? C'était un militant à l'ancienne, un romantique qui faisait tout passer par sa plume, captait l'émotion prise sur le vif. C'est un univers, c'est un monde à lui seul! Il a été de chair et de sang, s'émouvant aisément, s'émerveillant de tout et témoignant tout le temps. Il a écrit constamment, a voulu raconter, décrire, transposer. Il a laissé des milliers de pages, faites de correspondances, de nouvelles, de notes, de pièces de théâtre, et même de ses mémoires. Il impressionnait ses amis par ses humeurs, sa fantaisie et, surtout, par ce charme si particulier qu'il émanait.

Fallait-il commencer par l'œuvre qui l'avait fait entrer dans la postérité ou bien, à l'inverse, par tout ce qu'on ignorait de lui ? L'orientation de la biographie pouvait se faire, très simplement, par le choix de son nom. Elle aurait ainsi suivi la voie chronologique des vies qu'il avait successivement endossées: Semyon Akimovitch, Z. Sinnani, ou encore Shloymé An-Sky. Mais, entre le militant révolutionnaire Salomon Rappaport, l'homme de la mine Akimovitch, l'obscur écrivain Sinnani, le monument de la littérature yiddish An-Sky, quel pouvait être le dénominateur commun? Certes, chacun de ces noms présentait une facette du même homme qui le plaçait dans un cadre historique précis, éclairant d'un coup de projecteur un aspect singulier des tribulations juives et russes de la fin du XIX ${ }^{e}$ siècle et de l'aube du XXe. Le dénominateur existe pourtant bel et bien : le héros est, a toujours été, un écrivain. Catalogué parmi les auteurs patrimoniaux de la langue russe de l'ancien régime, ce sont ses noms de plume qui l'ont soustrait à l'anonymat, et ces mêmes pseudonymes signalent la teneur successive des œuvres qu'il a produites. Un second dénominateur pouvait être fourni par son engagement permanent dans l'action. Sociale, politique, ethnographique ou littéraire, elle semble avoir été le signe distinctif d'un homme toujours en mouvement, constamment dissimulé sous la cause qu’il défendait. Enfin, il était une autre caractéristique plus personnelle: quelque époque que ce soit durant sa vie, le héros ne s'était jamais installé, il avait toujours habité l'errance, n'avait jamais pu s'établir en aucun lieu, investir aucun logis, comme une âme toujours nouvelle qui viendrait habiter un corps se trouvant toujours ailleurs.

\section{D'un monde à l'autre, une biographie en paradigme}

Lorsqu'il naît, le 15 octobre du calendrier julien de l'année 1863, à Sczaznick, une bourgade de Russie blanche, ses parents Aron et Anna Rappaport le nomment simplement Shloymé (Salomon) Zanvl (Zangwill). Un père courtier en denrées et services divers, toujours sur 
les routes, une mère tenancière de pauvre taverne, deux sœurs : tel est son milieu familial. Une enfance de jeune garçon juif dans la zone de résidence, où l'on rêve plus qu'on ne joue, où l'on en apprend plus sur la dureté de la vie quotidienne que dans aucune école; où l'on attend, avec fièvre, un avenir meilleur, dont on sait à l'avance qu'il faudra lutter pour l'obtenir. Le héros a probablement grandi avec pour horizon la certitude qu'un sort individuel ne saurait être amélioré que par l'obtention de meilleures conditions pour tous. Cette ligne de départ peut tracer un scénario exemplaire, illustrant nombre de parcours de jeunes juifs de cette fin XIX siècle qui placèrent le meilleur d'eux-mêmes dans l'espoir de la réalisation d'un idéal social, passant par la révolution.

Cette ligne demeure cependant lacunaire. Elle ne pourrait rendre qu'une infime partie de la complexité de la personne qui a endossé tant de vies différentes. Une autre option serait la conflagration déterminante du contexte historique dans la vie du héros. Les différentes étapes de l'histoire politique russe introduisent, en effet, des scansions nettes dans sa ligne biographique. L'errance de Salomon Rappaport commence dès l'âge de dix-sept ans, alors qu'il passe d'une ville à l'autre à l'intérieur de la zone de résidence, surtout à l'intérieur et aux environs de sa province de Vitebsk (1881-1885), avant d'entamer ses pérégrinations subreptices hors de la zone réservée aux juifs. Les années suivantes seront jalonnées par des séjours plus ou moins prolongés qui l'éloigneront autant de sa province natale que des lieux de vie juifs. La région de Moscou d'abord, puis les mines de charbon et de sel dans l'Oural accueillent Semyon Akimovitch. À partir de 1891, ce sont les environs de Saint-Pétersbourg qui marquent l'entrée timide de S. Vid'bin, Z. Sinnani et Shloymé A. An-Sky dans les milieux de l'intelligentsia et au sein de la mouvance populiste. Salomon Rappaport délaisse la Russie tsariste, peut-être poursuivi, menacé d'emprisonnement et de déportation pour ses activités politiques, ou bien simplement laissé sans protection par la maladie de son protecteur, Gleb Ouspenski. Comme tant d'autres, il transite par Berlin, d'où il pousse jusqu'à Berne avant de s'établir à Paris où il restera jusqu'en 1905. Secrétaire particulier de Piotr (Pierre) Lavrov (de 1894 à 1900), ces années parisiennes sont marquées par ses activités de militant tous azimuts.

L'amnistie de 1905 permet à Rappaport de rentrer en Russie. Dans ses carnets se trouve Di shvoue, le serment, devenu l'hymne du mouvement nationaliste juif, Bund, mais aussi la traduction yiddish de l'Internationale entonnée désormais par toutes les générations de communistes et révolutionnaires juifs qui se succéderont. En avril 1906, il participe au congrès du Parti socialiste-révolutionnaire (SR) qui se tient à Vitebsk et démarre, simultanément, une tournée générale des lieux où se sont 
déroulés les pogroms qui ont jalonné l'effervescence de la révolution manquée, tout en assistant aux réunions du parlement nouvellement institué, la Douma. Lorsque la guerre éclate en 1914, il dirige une expédition ethnographique sur les juifs de la zone de résidence, qu'il poursuivra jusqu'en 1916, tout en s'enrôlant dans l'EKOPO, le comité d'aide aux victimes de la guerre. Après la révolution d'Octobre, il représente Petrograd à la Douma. Éminent membre des SR, opposants notoires au régime institué par les bolcheviques, il fuit en 1918 vers Vilno. Il meurt à Varsovie le 8 novembre 1920.

Cette ligne, si elle ne manque pas d'intérêt, reste cependant bien frêle au regard de l'entrelacement de ses productions littéraires et de sa vie personnelle. Pourquoi ne pas s'engager alors dans une direction biographique qui suivrait celle de ses publications?

Comme nombre d'écrivains en herbe, Rappaport entame une carrière littéraire incertaine où les fins de non-recevoir voisinent avec quelques publications. Il écrit des nouvelles réalistes en yiddish, parfois traduites en russe. Entre 1882 et 1886, ce sont surtout les situations cocasses et les relations qui se nouent entre les gens qui peuplent la zone de résidence qui irriguent son inspiration. Se succèdent ainsi des portraits de familles, de tavernes, des épisodes croqués de la vie quotidienne. Lorsqu'il quitte la zone de résidence pour s'enfoncer dans la vie russe, ce sont les paysans et les mineurs dont il cherchera à capter les traits et à rendre des tranches de vie. Se muant en plumitif populiste, il trouve dans la vie rurale et les caractères de l'insondable pauvreté russe, de quoi nourrir sa quête. Abandonnant l'écriture confinée à sa langue maternelle, c'est en russe que ses écrits seront dorénavant publiés. À partir de 1889, ses accointances, timides d'abord, puis ostensiblement affichées, avec les membres de l'intelligentsia lui ouvrent les portes des grands journaux, et ses chroniques, pièces et nouvelles paraissent quasi régulièrement. Son séjour en Europe parmi les autres exilés élargit le champ de ses horizons. Ses rencontres avec la littérature et les militants européens, notamment français, l'inciteront à nommer différemment ses aspirations d'homme de lettres. Du trait capturé sur le vif, de la description naturaliste de la vie quotidienne dans lesquels il trempait jusque-là sa plume, il va se porter vers la saisie de l'intérieur de caractères qu'il souhaite dépeindre, et c'est à partir de ce qu'on appelle alors le folklore que Rappaport va puiser ses formes littéraires. À cet intérêt pour la culture populaire, qu'il entend comme le génie des sociétés, s'ajoute une ambition théorique, qui inspirera la rédaction d'essais: L'Art populaire juif (1908), Qu'est-ce que l'anarchisme? (1907), Le Peuple et le Livre (1894) en sont quelques exemples. 
Les Euvres complètes en yiddish d'An-Sky (cf. 1920-1928), publiées à partir de 1920, après son décès, à Varsovie, rassemblent des écrits disparates rédigés au long de sa vie. Souvenirs, poésies, nouvelles, pièces de théâtre côtoient des témoignages et des essais, parfois traduits du russe parfois directement écrits en yiddish. Il est remarquable qu'une fois passé de l'écriture en yiddish, sa langue maternelle, au russe, c'est à cette dernière qu'il confiera le plus volontiers sa plume; plume qui demeurera son meilleur moyen de gagner sa vie. Ce sont, en effet, surtout les chroniques envoyées aux journaux qui parviennent tant soit peu à le nourrir, tout en lui permettant de vivre librement en tant que témoin engagé. Marquant sa période française, l'essai sur Émile Zola - dans les archives de Kiev - signale également le choc produit sur An-Sky par l'affaire Dreyfus et son traitement dans la presse, notamment les caricatures qui paraissent alors quotidiennement. Ses réflexions sur les rues et les cafés parisiens expriment l'attraction que la ville lumière exerce sur lui, tandis que le sort des paysans et des ouvriers reste dans sa ligne de mire, en lui permettant d'amorcer une étude comparée des conditions de vie des petites gens, dans l'empire russe et en Europe. Exilé politique à Paris, les moindres déplacements du trublion sont soigneusement relevés et garnissent les dossiers de l'Okhrana. Secrétaire de l'Internationale en titre, il rencontre ainsi régulièrement des groupes de révolutionnaires, parfois juifs, dans les sous-sols et les caves des cafés de rendez-vous, mais il visite régulièrement les pays alentours, rentre même clandestinement chez les siens, histoire de respirer un peu l'air du pays. Il s'agite beaucoup en Suisse, où ses amis les plus proches passent le temps, en manifestations diverses, politiques, certes, mais aussi en spectacles et amusements de toutes sortes qui emplissent leur quotidien. C'est encore en tant que journaliste, qu'il peut rentrer en Russie, afin de rendre compte dans la presse internationale des exactions commises à l'encontre des juifs en 1905-1906, puis du procès Beilis, cette accusation de meurtre rituel qui défraya la chronique en 1911 en réveillant les démons médiévaux. Il serait tentant alors de comparer ses écrits avec ceux de ses contemporains militants et théoriciens, de les remettre dans ce contexte et d'explorer la géographie des colonies russes en exil.

Cette ligne biographique oriente la description d'une vie de militant alliant la plume à l'action qui rendrait assez justement le caractère du héros. Elle pèche pourtant par une banalité à laquelle sa personne ne plie pas. Car il existe une singularité An-Sky, qui transcende ses actes et son accomplissement littéraire, et ne le situe ni au côté d'un Ravachol ni à celui d'un Tolstoï. Il faut en convenir : à mes premières rencontres, 
je n'avais pas la moindre idée de cette singularité. J'avais beau avoir un peu lu ses œuvres, la fascination que ses écrits parvenaient à exercer me demeurait un peu opaque. Le dybbouk faisait partie de mon héritage, en tout cas je le considérais comme tel, et, en tant qu'historienne en devenir, la description que An-Sky faisait de la destruction de l'ancien monde juif pendant la Première Guerre mondiale me paraissait essentielle et par trop négligée, remisée au placard par la Shoah. Et la singularité de cet homme ne m’a envahie, telle l'ombre sortie du passé, que lorsque j'ai commencé à vraiment chasser sur ses terres.

\section{La faille temporelle}

J'ai atterri en URSS sans connaître les tours et détours de l'univers soviétique. Je n’imaginais rien des arcanes que la bureaucratie et les difficultés quotidiennes pouvaient poser comme embûches aux chercheurs. Invitée officielle de l'Académie des sciences, j'étais accompagnée dans tous mes déplacements, reçue dans toutes les archives, traînée dans tous les opéras, mais ne connaissais personne. Je n'avais qu'un sésame: son nom. Pire encore, j'ignorais tout des lieux où les archives des militants antibolcheviques avaient pu être déposées. Je savais seulement que l'on venait de permettre aux chercheurs étrangers de venir s'y plonger. Par le jeu de multiples hasards et de beaucoup de chance, son nom a fini par résonner auprès d'inconnus avec lesquels je me liai immédiatement. Ces inconnus le connaissaient bien : il était leur guide sur le parcours d'un retour vers la judéité. Hormis le questionnaire publié en 1914 que j'avais eu sous les yeux, rien ou presque n'avait pu être vraiment édité des résultats de l'expédition ethnographique menée dans la zone de résidence. La guerre d'abord, la débâcle tsariste ensuite, enfin la fuite et les errances d'An-Sky, suivies du démantèlement des organismes et sociétés savantes de l'ancien régime, puis du passage à la trappe de tout ce qui était juif, avaient soit fait disparaître les précieux carnets, notes, dessins et objets collectés, soit tout simplement mis sous le boisseau ce qui en pouvait rester. Mais il existait un volume de souvenirs de l'un des membres de cette expédition : publié par Abraham Rechtman dans la communauté yiddishiste argentine en 1958, ce volume, dédié à la mémoire de Shloymé Zanvl fils d'Aron le Cohen Rappaport, Shloymé An-Sky, est un tribut offert à l'expédition ethnographique dont il ne reste rien. Rechtman, l'un de ses rares survivants, y reporte fidèlement ce que sa mémoire a pu retenir des circonstances qui ont mené à cette entreprise : les itinéraires choisis, les lieux visités, les méthodes employées. Il décrit les événements dont lui et ses coéquipiers ont été témoins, et rappelle certains des résultats obtenus, sous forme de récits ou de comptes rendus. Plus qu'un livre 
pour mémoire, comme en ont publié en nombre les survivants des communautés décimées par la Shoah, ce livre présente une somme inachevée d'instantanés, de rencontres et de récits rapportés sur le vif et retranscrits quelques décennies plus tard.

Curieusement, c'est par l'intermédiaire du livre de Rechtman, utilisé pour l'apprentissage du yiddish, que ces inconnus avaient rencontré An-Sky et que celui-ci était devenu leur maître en judaïsme, celui qui ouvrait la voie et dirigeait leurs pas vers la redécouverte de leurs origines, de leur patrimoine dissimulé, perdu, oublié depuis quatre-vingts ans. Nous communiquions plus par signes et par bribes que par un véritable échange dans une langue commune, mais l'enthousiasme pour le même héros aidant, nous nous comprenions parfaitement. C'est alors qu'une tout autre orientation biographique s'est frayée une voie.

An-Sky, ressurgissant sous l'effet boomerang d'une histoire se faisant régressive, ne se contentait plus d'appartenir à son temps, d'incarner une sorte de paradigme d'un monde et d'un milieu désormais évanouis. Il existait dans le temps présent, tel le personnage auquel il avait prêté sa plume: l'émissaire des autres mondes, qui transcendait les univers et enjambait les barrières du temps. Il fallait donc duper le démon, et s'emparer du dybbouk, au lieu de lui donner prise. Suivre ce fil pouvait permettre de dépasser le genre biographique classique, afin de tenter d'en faire un mélange exemplaire: une vie et une œuvre mêlées dans l'histoire, se rejouant entre passé et présent.

Au temps présent, renouant avec les projets ébauchés par les intellectuels juifs du début du siècle, des chercheurs novices commencèrent à édifier des réseaux qui, de la Russie à la Lituanie en passant par l'Ukraine, conçurent de passer au peigne fin tous les instituts et centres d'archives dans lesquels des documents concernant les juifs avaient pu être entreposés au fil des ans. L'entreprise n'était pas chose facile. Aux problèmes de paperasserie administrative s'ajoutait également celui de la mise au secret des documents: depuis les années 1930, la plus grande partie des documents concernant les juifs et leurs activités avait été scellée. La libéralisation progressive du régime, les possibilités de libre circulation des personnes inspirèrent de nouvelles actions. Des groupes, clandestins d'abord, constitués ensuite légalement dans les universités, établirent des programmes d'investigation autour des centres séculaires de la vie juive en Russie. Reprenant toutes les descriptions existantes de villes et villages habités autrefois par des juifs, rassemblant des photographies, des cartes postales ou des textes, ils avaient commencé à instaurer des «programmes de vacances " destinés à parcourir ces régions en vue d'y recenser toutes 
les marques encore identifiables de la vie juive d'antan. À l'inventaire des villages et des cimetières juifs, parfois détruits et profanés, ils ajoutèrent les synagogues détournées, abandonnées ou rasées, les instituts historiques des provinces pour en analyser les archives; mais ils souhaitaient également retrouver des habitants juifs dans les anciennes bourgades, rassembler leurs récits, reconstituer leur mémoire et leurs histoires. Pour certains de ceux que je rencontrai, le projet était de quitter ce qui était encore l'URSS en emportant des fragments du patrimoine ancestral, qui permettraient de reconstituer les éléments d'une documentation historique utilisable et transportable vers l'Occident. Pour d'autres, il s'agissait de retrouver des racines profondément enfouies, afin de reprendre le cours d'une histoire interrompue.

Les chercheurs néophytes durent ainsi apprendre sur le tas, par l'entremise des résultats inaboutis de l'expédition ethnographique stoppée en 1914, les méthodes souvent rudimentaires qu'ils allaient utiliser. Sous l'ancien régime, les juifs interdits dans les villes et capitales ne pouvaient pas choisir leurs lieux d'habitation, ils étaient massivement concentrés dans le "rayon", la zone de résidence constituée par les régions dans lesquelles ils vivaient, prises lors des partages de la Pologne (Lituanie, Biélorussie, Bessarabie, Podolie, Volhynie) et auxquelles furent ajoutés le sud de l'Ukraine et les rives de la mer Noire. Les premières années de la Révolution leur donnèrent la possibilité de pénétrer dans les grandes villes, mais la libre circulation des personnes et les déménagements spontanés n’acquirent jamais vraiment droit de cité dans le bloc soviétique. Les "années Gorbatchev" rendirent possibles les déambulations, sac au dos, et l'ancienne zone de résidence devint le lieu d'investigation privilégié par ces « vacanciers » dont le nombre croissait, passant en quelques années d'un cénacle de six personnes à des expéditions de cinq ou six groupes différents. Afin de déchiffrer ce qu'être juif pouvait bien signifier, ces jeunes russes découvraient les vestiges d'un monde non seulement englouti, mais dont ils ne savaient rien. Maints hameaux, nombre de bourgades et bien des villes de la zone de résidence avaient abrité des populations juives depuis le Moyen Âge ; certaines localités ayant été entièrement édifiées par des juifs ne comportaient même pas d'église et on leur raconta que beaucoup d'enfants ukrainiens d'une époque révolue n'avaient dû leur instruction élémentaire qu'au fait d'avoir été scolarisé dans les écoles juives du lieu. Même si, durant les années qui suivirent la Révolution, des communautés et des familles s'étaient maintenues sur place, la plupart de ces villages offraient, à l'aube des années 1990, la vision d'un univers juif fantomatique qui en laissait percevoir la dévastation. Ces régions, autrefois peuplées d'une manière si dense, 
furent la cible de toutes les exactions militaires qui ravagèrent le siècle : zones de combat et de front pendant la Première Guerre mondiale, les populations en avaient été maintes fois déplacées, et les pogroms qui s'y déroulèrent durant la guerre civile en modifièrent profondément la structure démographique. Enfin, les massacres perpétrés par les nazis, dès les premières heures de leur arrivée, achevèrent de décimer les communautés restées sur place. Les survivants, de retour, choisirent pour beaucoup de tenter leur chance ailleurs. De sorte qu'en même temps qu'ils découvraient une archéologie du monde juif, ces groupes rencontraient les atrocités d'une histoire qui n'avait pas été vraiment évoquée ni transmise par les voies officielles. Cette confrontation était loin d'être plaisante. Souvent, lorsqu'ils demandaient où trouver le vieux cimetière juif, on les conduisait sur la place d'un massacre abominable et la recherche des tombes dans des champs en friche, qui en faisaient une jungle inexplorée, amenait les paysans du lieu à faire un récit insoutenable pour leurs auditeurs, dans lequel se mêlaient indistinctement les anecdotes nostalgiques d'un autre temps à celles de la barbarie nue. Ces premières années de recherche consistèrent ainsi en la recomposition d'un puzzle tant historique que géographique et affectif. Ce puzzle devait aboutir, dans une étape ultérieure, à la constitution de quantité de savoirs nécessaires à la collecte des données et, enfin, servir à la réécriture d'une histoire des juifs en URSS, occultée par les instances académiques officielles et par l'absence de contacts avec l'extérieur. Pour rassembler les données, il leur avait fallu se familiariser à la fois avec les langues juives et avec celles des régions visées (yiddish, ukrainien, lituanien, etc.), mais aussi mettre au point des méthodes de travail pour lesquelles aucun d'entre eux n'avait reçu de formation initiale. La plupart des membres de ces groupes apprirent les bases élémentaires linguistiques par leurs propres moyens, les dossiers d'apprentissage mis en circulation en URSS par l'Agence juive ne portant que sur l'hébreu. Pour le reste, la littérature et l'enthousiasme servirent de modèle et de règle. Le plus difficile à monter fut peut-être - comme toujours en URSS - la partie technique. Trouver du matériel photographique (appareils, pellicules et papiers) et sonore (magnétophone et cassettes), récupérer des cartes régionales (denrée ultra-secrète) représentaient peut-être les opérations les plus compliquées à effectuer. Les premières expéditions, lancées en 1988 et 1989, s'étaient déroulées dans une ambiance de découverte et d'exaltation. D'anciens cimetières juifs, localisés par les indications données par de vieux autochtones, avaient été exhumés à la serpe; des pierres tombales séculaires avaient été déterrées, qu'il avait fallu défricher et gratter durant des heures; de vénérables synagogues, mentionnées dans quelque écrit, avaient été identifiées, 
transformées en magasin de brique ou en silo à grains. Mais, il leur avait fallu également parvenir à mettre suffisamment en confiance quelques "anciens» pour qu'ils partagent ce dont leur mémoire était encore le réceptacle : proverbes, chansons, anecdotes... Pourtant rien n'était joué puisque tous les éléments de l'arrière-fond manquaient encore à ces chercheurs en herbe pour agencer et analyser leurs travaux : quelle avait été l'importance des communautés dont subsistaient les vestiges; évaluer la renommée ou l'anonymat des personnes reposant sous les tombes; connaître la notoriété des maîtres ou des rabbins locaux.

Pratiquement coupés de l'extérieur pendant quatre-vingts ans, beaucoup parmi les juifs russes ont longtemps ignoré les canaux de la transmission du judaïsme devenus usuels dans la société occidentale contemporaine. L'observance des préceptes et des lois, ou même l'identité culturelle diffuse jusque dans la laïcité par des voies et des attachements divers se sont maintenues d'une manière qui ne va pas sans évoquer celle des marranes en d'autres temps. L'inscription d'une "nationalité" juive affichée sur les papiers officiels, parfois embarrassante, réduisant les possibilités d'insertions sociales et professionnelles, n'en était que l'un des aspects ambigus, lorsque, comme souvent, elle s'accompagnait de l'absence de tout sentiment d'appartenance à un groupe.

Cependant, la guerre de juin 1967 produisit, en URSS comme ailleurs, un fort sursaut identitaire, exprimé par une marée de demandes d'émigration, appuyée par les campagnes internationales des années 1970 en vue d'obtenir la « libération des juifs d'URSS ». Une volonté d'identification à l'État d'Israël valeureux et conspué chez eux fut, peut-être un temps, le signe d'un événement survenu dans les consciences des juifs d'URSS, dans lequel il n'est pas aisé de mesurer combien a pesé la part des difficultés inhérentes au système communiste et à une forme d'antisémitisme perdurant. Mais, lorsque les portes commencèrent à s'entrouvrir et que les premiers immigrés témoignèrent des innombrables problèmes qu'ils rencontraient quotidiennement en Terre promise, les perspectives et les horizons se transformèrent. Les échanges et les contacts se multipliant sous toutes les formes, nombre de juifs d'origine russe prirent soudainement conscience de l'abîme qui les séparait de leurs coreligionnaires occidentaux et orientaux. Quatre générations d'interdits religieux avaient rendu la plupart d'entre eux imperméables à une pratique dont ils ne pouvaient ni apprécier les contraintes ni comprendre les restrictions. La transmission familiale des uns et des autres pouvait se fonder sur l'idéal communiste pour lequel on avait combattu depuis toujours, mais aussi sur le souvenir de l'aïeul dont on racontait pieusement qu'il ou elle avait conservé jusqu'à la mort, et dans l'illégalité la plus dangereuse, la langue 
et les traditions ancestrales. D'autres encore n'avaient ni récit d'hérö̈sme ni souvenir d'anciens. En ce sens, l'ouverture d'universités juives ou de départements d'études juives, aujourd'hui officiellement reconnus et bénéficiant d'un véritable statut académique à Moscou ou Saint-Pétersbourg, offrait, en même temps qu'une projection sur l'avenir, une rencontre avec les vestiges d'un passé et la reconstitution d'une histoire commune. Mais cette démarche impliquait aussi la mise à jour d'une culture dont personne ne détenait plus les clés. De sorte qu'en s'ajoutant aux cours sur le judaïsme et son histoire, qui pouvaient donner sens à une "nationalité » estampillée sur des papiers d'identité, les expéditions et les investigations d'archives permettaient de renouer avec un passé occulté, destiné à être transmis désormais par la réunion, dans une mémoire collective entièrement reconstruite, des débris épars d'une culture et d'une histoire spécifiques aux juifs russes.

Entre passé et présent, s'agençait donc, subrepticement, une pièce d'histoire, dont la rédaction avait été ébauchée près d'un siècle auparavant. Le projet de construction de l'identité juive par le biais de l'approche scientifique avait déjà été tenté dans le judaïsme allemand au XIX siècle. Il s'agissait alors d'édifier une judéité plus intellectuelle qu'émotionnelle, pouvant rivaliser avec les formes chrétiennes de la religion moderne. Dans la sphère orientale de l'Europe, pour An-Sky et les siens, la démarche identitaire avait été autre, et il l'avait affirmée avec hauteur: «les juifs de l'intelligentsia n'ont aucune connaissance [du judaïsme], quelques expressions et quelques blagues leur suffisent pour se considérer comme des experts es Judentums" (in 1920-1928 : XV, 30). Certes, pour lui, il n'était pas pensable de passer outre l'amélioration de tous et d'ignorer le passage obligé d'une révolution politique qui délivrerait les peuples de la servitude, mais, simultanément, la conscience aiguë d'un trésor culturel gisant dans le «folklore» populaire affermissait un souci de préservation - ou d'embaumement - de ce qui faisait la spécificité de l'être juif.

De sorte que, par la singulière ironie que l'histoire sait parfois réserver, le phénomène de la quête de l'identité juive s'était inversé avec l'assimilation des juifs dans l'environnement: en Russie, par exemple, l'antisémitisme avait parfois été le seul indicateur de judéité, et le recours, grâce à la médiation d'un savoir académique, à la reconstitution d'une mémoire non transmise pouvait paraître efficace. Mais cette judéité se construisait aussi, et peut-être surtout, comme l'expression précieuse de la liberté recouvrée. 


\section{L'homme d'entre les mondes}

Près de vingt années se sont écoulées depuis ce cheminement à tâtons entre la figure du passé et sa trace dans le présent. Le présent d'alors s'est, entre-temps, lui aussi enfoncé dans le passé, et An-Sky a pénétré l'univers statique des figures de paradigmes historiques. La possibilité d'accéder aux archives autrefois interdites lui a fait quitter l'ombre pour se muer en proie. La correspondance monumentale qu'il avait échangée avec tous ses contemporains a trouvé un vaste rang de lecteurs et, plus qu'aucun autre personnage, il est entré dans la légende sous le masque du héros, illustré par l'article séminal de David Roskies (1992). Parangon du judaïsme russe de la fin du XIX siècle, c'est autour de lui que David Biale fait graviter son chapitre sur l'Europe de l'Est dans la somme des Cultures des juifs. Une nouvelle histoire (2002 et 2005). Source constante d'inspiration, ses nouvelles, traduites en anglais, forment à présent un véritable fonds culturel où vont puiser les romanciers et gens de cinéma qui veulent conférer une forme de réalité au passé juif. Les musiques et cylindres rassemblés lors de l'expédition ethnographique et reportés par les grands musicologues de l'époque, Iuli Engel (1868-1939) et Zinovy Kiselhoff (1878-1927), approvisionnent les musiciens klezmers, tandis que les croquis effectués par Shloymé Youdobin ont été immortalisés par les artistes juifs, dont Chagall a été le plus représentatif. Une exposition intitulée "Sur les traces d'An-Sky» (Beukers \& Waale 1992) a traversé le monde; un gros volume, The Worlds of S. An-Sky (Safran \& Zipperstein 2006) traquant toutes les facettes de sa vie et de ses œuvres au prisme des études réalisées par dix-neuf chercheurs, est paru, comportant de surcroît une chronologie aussi fiable que possible de ses errances et de ses rencontres. Et encore faut-il ajouter à cela une succession d'articles épars, de numéros spéciaux de revues, de publications d'extraits choisis parmi ses œuvres complètes. De chaque témoignage, de chaque article produit, émerge un aspect singulier d'An-Sky : poète, musicien, inspiré par les femmes, prolétaire, politique, utopiste; homme de lettres, ethnologue, muséologue, mystique, révolutionnaire, masqué. Et bien qu’on puisse concevoir que chaque étude construise sa propre figure d'An-Sky, en l'adaptant à une démarche historique ou littéraire, il faut convenir que la plupart des auteurs prennent pour acquis la figure d'un homme déchiré entre des idées et des désirs contradictoires ou encore celle d'une vie composée de deux parties distinctes. Cette reconstruction de cet homme infiniment public sait ainsi rassembler les rêves des chercheurs autour d'une image qui se recompose perpétuellement, et forme un puzzle où manqueront toujours quelques pièces. Car, malgré l'abondance de 
témoignages, de lettres et d'écrits laissés à la postérité, l'homme qui se tenait derrière la plume persiste à échapper à ces tentatives de captation posthume en dépit de la dimension imposante de la statue mémorielle qui lui a été édifiée.

La toute première statuaire fut édifiée par ses proches. "L'ami de toute une vie", rencontré à l'âge des jeux dans les cours et sur les places de Vitebsk, Chaïm Zhitlovsky (1861-1943), s'étant attribué la fonction d'exécuteur testamentaire et de biographe officiel, en a façonné la première ébauche dès après sa mort, en 1920 (cf. 1935). Les orientations politiques les ont séparés assez tôt, mais les liens affectifs qu’ils avaient tissés compensaient leurs différents idéologiques. Au fil d'une correspondance et de retrouvailles régulières interrompues seulement par la mort d'An-Sky, les deux amis ont toujours partagé leurs expériences. Issu d'une famille aisée, Zhitlovsky avait effectué des études supérieures; philosophe de formation, son nom est surtout resté attaché à ses œuvres en tant que théoricien marxiste du nationalisme juif. Jadis, dans leur prime jeunesse, ils avaient cheminé d'un même pas, et participé tous deux activement au Parti SR. Mais, Zhitlovsky s'était rapidement investi dans la cause du sort des juifs, tandis que Rappaport demeurait engagé dans un populisme moins spécifiquement orienté. Zhitlovsky, en tant qu'ami de toujours et en biographe légitime, a esquissé la trajectoire d'An-Sky par la métaphore de l'aller-retour. Le jeune Rappaport se serait ainsi délibérément détaché de la maison juive, jusqu'à devenir un véritable goy, tout en restant néanmoins un "juste parmi les nations", tandis que l'adulte, An-Sky, aurait parcouru le chemin en sens inverse, devenant un pénitent accompli vers la fin de sa vie en rejoignant le bercail de la yiddishkeit, la judéité. Cette version de vies parallèles a depuis lors retenti sur tous les écrits qui ont été consacrés à An-Sky. Ce schéma est adopté par Victor Chernov (1948), pilier du Parti SR, qui dédie un chapitre de sa tournée des dirigeants juifs du parti à Semyon Akimovitch An-Sky. Ils s'étaient rencontrés chez Piotr Lavrov et une solide amitié, entretenue par une correspondance suivie, s'est développée entre les deux hommes. Ajoutant au portrait tracé, via les anecdotes racontées par An-Sky lui-même, les éléments biographiques manquants puisés chez Zhitlovsky, il paracheva, de la sorte, cette image. En le présentant comme un homme chaleureux, avec un visage typiquement juif, le port un peu voûté et qui aurait eu, s'il n'avait été glabre, l'allure "majestueuse d'un prophète", il rejoint celui tracé également par nombre de ses autres amis. Tous rappellent ce mélange subtil de légèreté et de gravité qui, selon eux, caractérisait An-Sky. Simon Doubnov, l'historien juif avec lequel An-Sky s'est souvent 
affronté autour d'idées, évoque dans son journal la figure d'un perpetuum mobile, un éternel errant se consumant en de multiples activités: journaliste, ethnographe, militant et militaire, passant du groupe des nationalistes au camp sioniste, puis des rangs des cadets vers sa famille du Parti SR (Doubnov 2001 : 912).

Curieusement, pour un personnage dont la vie est tellement documentée, la période précédant l'entrée de Rappaport dans l'intelligentsia juive n'a guère laissé de traces. Ce que l'on sait tient à peu de chose : ses premiers engagements en tant que précepteur particulier dans les foyers juifs aisés de sa province, dissimulant l'enseignement à la sédition sous le couvert du religieux, son passage dans les mines, quelques images de sa vie en commune, où quatre affamés se partageaient une paire de chaussures et un manteau... La foisonnante quantité de témoignages dont on dispose provient de ceux qui l'ont rencontré alors qu'il était déjà devenu un membre éminent de la rue juive. Ses écrits, demeurés en bonne place dans les anthologies de la littérature russe puis retravaillés pour être traduits en yiddish dans ses œuvres complètes, sont cependant pour nombre d'entre eux des récits biographiques à peine transposés. Depuis son adolescence, An-Sky remplissait des carnets où il notait méticuleusement ses remarques, ses impressions, et dans lesquels s'esquissaient des ébauches de nouvelles, de poésies, des portraits, il y déposait des anecdotes aussi bien que des proverbes. Ses cahiers, véritables carnets de bord, ont été le fonds d'où il puisait son inspiration et avec lesquels il étoffait les trames de ses écrits. Sa vision d'une vie à habiter comme une œuvre transpire de tous ses actes, de tous ses écrits, qu'il s'agisse de ses lettres ou de sa littérature ou encore de ses essais. En fidèle Narodnik, il entendait le rôle de l'intellectuel comme celui d'un guide, ou d'un éducateur. Et, comme il l'avait déclaré avec emphase dans l'une de ses lettres de jeunesse à Zhitlovsky, il fallait savoir se départir de sa famille, de ses proches et des siens pour mieux exercer cette mission (Krutikov 1994). Car mission, il devait forcément y avoir. An-Sky n'imaginait pas une vie qui ne serait pas vouée à une cause, et tant qu'à faire, il fallait trouver la plus juste, la plus fervente, celle qui permettrait d'embraser et de combler une existence entière. Ainsi ses errances, loin de marquer des égarements, attestent-elles simplement la multiplicité des aspirations d'un rêveur, additionné d'un irréductible romantique. Écrire pour militer, militer pour écrire, et vivre une vie digne d'être racontée aurait pu être sa devise. Il comprenait le folklore, tel qu'il s'était révélé à lui durant ses années parisiennes, comme les fondations de la culture ou plutôt comme l'âme d'un peuple. 


\section{Le dybbouk dans l'histoire est caché}

La relation de Rappaport/An-Sky à sa yiddishkeit, son être juif, est probablement ce qui a fait couler l'encre la plus abondante depuis sa déclaration d'homme partagé, lors d'un dîner donné en son honneur dans un restaurant de Saint-Pétersbourg, à l'occasion de la célébration de son demi-jubilé littéraire qui accompagnait la sortie de ses premières œuvres complètes, en 1909 :

«Le sort de l'écrivain est généralement pénible, celui de l'écrivain juif l'est tout particulièrement. Son écriture est déchirée ; il vit dans deux rues, s'exprime en trois langues, vit misérablement en marge de toutes, et j'ai vraiment enduré ce fardeau. Lorsqu'il y a vingt-cinq ans je me suis lancé pour la première fois dans la littérature, l'aspiration d'œuvrer pour le bien des opprimés, des masses ouvrières, s'est éveillée en moi, et il me semblait alors - et là était mon erreur - que je ne pourrais la réaliser parmi les juifs. Je pensais qu'il était indispensable de tenir une position politique et là encore je ne trouvais pas dans le monde juif la moindre force politique qui détienne une réelle aspiration pour la judéité ; je me jetais alors de tous côtés et me suis porté vers un autre peuple. Ma vie s'est alors brisée, elle s'est écartelée, elle resta déchirée.

De nombreuses années de ma vie se sont déroulées dans ces marges, au bord de la séparation entre ces deux rues - et c'est pourquoi je vous prie de retirer seize années des vingt-cinq qu’a duré ma carrière littéraire jusqu’ici " (in Zerubavel 1964 : 5).

S'ajoutant aux propos de Zhitlovsky, cette proclamation publique a toujours été prise pour un constat avéré par les amis, biographes et chercheurs qui se sont intéressés à sa personne. C'est sur cet arrière-plan théâtral que sa personnalité d'intellectuel russe complètement étranger à l'authentique tradition juive a été édifiée avec d'autant plus d'emphase qu'il avait laissé cette pièce, considérée comme l'apogée voire la substance de l'âme juive, Le Dybbouk. Peaufinant l'image d'une vie en deux phases, la légende (académique) en a tiré la conclusion qui s'imposait: An-Sky aurait découvert, en même temps que son "être juif", la tradition séculaire de ses pères lors de l'expédition ethnographique.

Cette approche sans nuance fait ainsi peu de cas du maintien constant de ses activités politiques dans le Parti SR, notamment de sa députation à la Douma en dépit de son "retour" vers la cause juive, tout comme elle ignore les aspects juifs de ses écrits valant autant lors de sa "première " période que lors de la "seconde». Car, outre la vieille rancœur de Zhitlovsky qui n'avait pu lui pardonner ni son investissement dans l'internationalisme ni sa tendresse jamais démentie pour les «forces réactionnaires ", autrement dit l'univers religieux, Rappaport était entièrement juif par son être au monde. Sa façon de n'être ni ici ni ailleurs, perpétuellement à la frontière de deux mondes comme il l'avait déclaré sur le mode du mélodrame, en est la meilleure attestation. Ayant abandonné 
son vivier, il s'était retrouvé tel un étranger où qu'il se soit posé : des mines de l'Oural à Saint-Pétersbourg, de Berne à Paris, de Moscou à Varsovie, il était constamment tiraillé entre ses impulsions, ses volontés et les missions qu'il s'était fixées. Mais comment croire qu'il ait pu devenir étranger à lui-même au point de devoir découvrir la réalité de la judéité lors d'une expédition ethnographique? Son habileté à avancer masqué, développée durant sa jeunesse, puis son passé d'activiste, caractérisent son usage du double langage modulable selon ses auditeurs : ainsi, tandis que ses conférences témoignent du discours d'un homme des Lumières, rationnel et pédagogue, ses récits portent l'empreinte d'un univers peuplé d'anges, de démons, de rabbins et sont pleins de la présence de Dieu et des forces surnaturelles. Zhitlovsky décrit un jeune Rappaport, auquel il a eu le privilège d'apprendre à lire le russe, haïssant son milieu et ses maîtres: "l'époque du héder ["l'école élémentaire"] fut la plus sombre de notre vie. Le héder ne pouvait nous insuffler aucun amour pour le peuple juif ni la vie juive, pas plus que la maison de prières, dont l'ambiance était infernale" (1935: 14-15); et il ajoute qu'on n'y apprenait pas grandchose, peut-être pas même à écrire correctement. Mais une tout autre musique se dégage du récit recueilli par Shmuel Leyb Zitron, un autre de ses amis :

"C'était un jour d'été, entre minha et mảariv ["au crépuscule, entre les prières de l'après-midi et du soir"], en une minute, je fus submergé par un océan de souvenirs d'enfance et de jeunesse. Je me suis rappelé notre bes midresh ["maison de prières"], où moi aussi, comme ces jeunes gens, je m'étais balancé avec exaltation et concentration sur la gemara ["page du Talmud"] et papa et maman qui bénissaient le rabbin que j'allais devenir. Je me suis représenté notre rabbin, tel qu'il était assis lors de la leçon et comme il discutait avec les érudits locaux qui avalaient littéralement chaque mot qui sortait de sa bouche. Un souvenir appelant l'autre, j’ai retrouvé nos shabes ["shabbat"] et yontoyvim ["jour de fêtes"], et les zemires ["chants liturgiques domestiques"] de papa, maman sous son foulard..." (cf. "Der Lezter fun di Drey [= Le dernier des trois]", in Shalit, ed. 1920-1921).

L'attitude des amis d'An-Sky à l'égard de sa phase d'éloignement du judaïsme peut néanmoins être appréhendée par le contexte politique de l'époque. La radicalité des positions idéologiques ne permettait pas, alors, de saisir l'aspect, devenu depuis de sens commun, de l'investissement typiquement juif dans l'internationalisme de cette fin de siècle, et le démon juif paraissait ne pouvoir se déployer que dans le nationalisme (juif) ou le sionisme, sous peine de trahir son camp. Or, An-Sky était de tous les combats, sensible à toutes les luttes sociales, culturelles et politiques. Cet éclectisme se manifestait aussi bien dans la quantité de sociétés savantes auxquelles il appartenait que dans la curiosité qui émane 
à tout propos de ses carnets. Alors que la conférence des langues, réunie à Czernovitz en 1908, consacre le yiddish comme la langue nationale juive, An-Sky défend le trilinguisme. Élevé, en yiddish, dans la tradition religieuse, ayant acquis la culture russe en autodidacte et plaçant aussi quelque espoir dans le rêve sioniste - le tout dans l'empire tsariste où la polyphonie des langues et des accents composait le fond sonore -, il n’imaginait probablement pas opportun de réduire la pensée et l'expression à un monolinguisme, fusse-t-il juif.

Le démon juif veillait cependant, attendant de reprendre la main sur le tour de l'histoire. Lorsqu'éclate l'affaire Dreyfus en 1894, Rappaport, fasciné, dévore la presse quotidienne, fulmine durant le procès, puis rédige son essai sur Zola, tout en s'interrogeant amèrement sur les vertus de l'assimilation et du progrès. Tout en représentant l'Internationale russe en exil, devenu secrétaire de Lavrov (incidemment l'une des rares grandes figures socialistes qui ait abordé les problèmes particuliers des juifs), An-Sky n'a jamais délaissé les contacts avec ses coreligionnaires, ouvriers ou artistes qui se trouvaient à Paris, ni interrompu ses échanges épistolaires avec ceux restés au pays, gens de lettres y compris. Plus encore, c'est au cours de sa période parisienne et européenne qu'il débute sa carrière de conférencier professionnel, traitant volontiers de la question juive dans l'empire tsariste, tout en fondant avec Chernov la Ligue socialiste agraire, embryon de ce qui allait former le Parti SR, appelant au combat pour la révolution en Russie. Un épisode rocambolesque l'allie au transfuge de l'Okhrana, le Père Gapone, dont on saura plus tard l'implication trouble dans le "dimanche sanglant" de Saint-Pétersbourg; misant sur sa renommée, Rappaport/An-Sky l'incite à rédiger un appel contre les pogroms qui se déchaînent dans le sillage des événements de 1905, mais, confondu et assassiné, son pamphlet restera lettre morte.

Cette période d'exil signale, pour qui accepte l'idée d'une vie fractionnée, l'inscription graduelle de la "judéité" d'An-Sky dans ses écrits comme dans ses activités. On peut néanmoins concevoir autrement cette accentuation d'expressions juives dans l'espace public. L'hypothèse d'une sorte de retour du refoulé pourrait aisément être justifiée par l'aggravation de la situation des juifs dans l'empire, d'une part. Mais, de l'autre, pourquoi ne pas considérer qu'An-Sky se soit simplement laissé porter par l'écho rencontré par sa plume? En suivant simultanément ces deux pistes, la linéarité historique et littéraire de la dernière décennie de la vie de Rappaport/An-Ski se déroule presque naturellement. 


\section{Revers biographique}

Revenons à la case départ. Né dans une famille pauvre, le jeune Rappaport décide d'échapper à un destin tracé dans la continuité de la tradition et des valeurs juives. Il s'engage, comme tant d'autres de sa génération, dans le mouvement juif qui combat pour l'émancipation. Sa rencontre en cours de route avec l'idéal révolutionnaire marque le déroulement d'une vie qui s'adapte au fur et à mesure des opportunités qu'elle présente. Espérant parvenir à vivre de sa plume, c'est en témoin engagé qu'il défend l'idéal auquel il se voue. N'ayant pas échappé à la précarité, le succès de sa carrière littéraire d'écrivain populiste étant resté tout relatif, c'est en tant que chroniqueur et journaliste qu'il subvient à ses nécessités élémentaires, la faim étant souvent le moteur essentiel de ses activités. Or, c'est dans le cercle de l'intelligentsia juive qu'il rencontre le plus d'échos et, en dépit de la multitude de ses liens avec l'univers non juif, c'est surtout celui de ses origines qui l'accueille. Il ne faut cependant pas minimiser l'importance de son être au monde. Juif par sa culture originelle, russe par aspiration, pour lui sa patrie est formée par la somme des deux qui demeurent «indissociables ». Mais son système de références, sa manière d'envisager la culture et la politique, et même d'investir une vie dans une ouvre, restent typiquement juifs. Il est absurde d'imaginer que c'est grâce à un travail artificiel d'écrivain que l'esprit de la tradition juive, incluant les caractéristiques de rythme et de genre des récits traditionnels, imprègne ses écrits. Mieux vaut prendre l'image de l'homme masqué, et plus précisément celle du marrane pour appréhender le personnage. C'est en effet en s'adressant depuis la rive extérieure à la maison juive qu'il parvient au mieux à faire entendre son discours de liberté, qu'il ancre, néanmoins, au plus profond de la tradition juive, la présentant comme du simple folklore. Toutefois, la notion de folklore qu'il éclaire dans l'introduction de son programme ethnographique dépasse largement le cadre de ses acceptions courantes:

" [...] à côté de la Torah écrite [toyre shebiktsav], nous avons également reçu l'héritage d'une autre toyre, une toyre shebalpe ["Torah orale"] que les gens, surtout les masses, n'ont cessé de créer au cours de l'histoire de leur longue, cruelle et tragique existence. Cette toyre shebalpe est faite d'histoires populaires et légendes, de proverbes et d'aphorismes, de chants et de mélodies, de coutumes, de croyances [...] en elle reposent la beauté et la grandeur de l'âme juive" (An-Sky 1914: 9).

D'ordinaire, cette distinction entre loi écrite et orale distingue le judaïsme biblique - la Torah écrite - du judaïsme rabbinique - la Torah orale, considérée comme l'ensemble de la doctrine talmudique, incluant tous ses commentaires et exégèses normatifs. Faisant du folklore la quintessence 
de l'âme juive, An-Sky n'effectue rien d'autre que couler la totalité de la vie dans la vaste doctrine du judaïsme. Et cette amplification même, loin d'en faire suspecter l'ignorance, permet, à l'inverse, d'identifier l'ampleur de l'ancrage d'An-Sky dans la tradition, qui entend englober la vie entière de l'individu dans ses normes. Son essai sur la création ethnopoétique juive publié en russe en 1908 est une première en matière de littératures populaires comparées. La Bible, l'Odyssée d'Homère, la Chanson des Nibelungen, ou encore la chanson de geste, doivent ainsi leur résonance universelle à leur enracinement dans ce qui est le plus impalpable de l'esprit des peuples, autrement dit, pour An-Sky, leur folklore.

Issu de la seconde génération des Lumières juives, Rappaport/An-Sky jugeait sévèrement ses résultats. Loin d'avoir su motiver l'émergence d'une nouvelle forme d'identité nationale, la Haskalah russe n'était parvenue qu'à provoquer le détachement des jeunes générations de leurs racines et à éveiller une forme de haine de soi, dont il avait directement fait l'expérience. Cette faillite de l'ambition lui paraissait être l'une des causes du délitement de l'espace juif. La rupture intervenue entre pères et fils signifiait la cessation de la transmission du passé dans le présent, et débouchait sur l'oblitération complète du legs amassé au long des âges: "emporté dans la tombe par l'ancienne génération" (1920-1928: $\mathrm{XV}, 32)$. Cet essai, qui anticipe l'érosion non seulement des traces matérielles, effacées comme toute chose par le temps qui passe, mais également de la mémoire collective, des traditions et des coutumes juives, marque l'irruption de la dernière vocation de la vie de l'homme d'entre les mondes : recueillir les vestiges de la tradition juive. Envisageait-il qu'elle puisse à nouveau pénétrer dans le cœur des futures générations? Voulaitil seulement en préserver le souvenir sous une forme muséale? Ou bien seulement pacifier avec le démon tapi dans la modernité ? Probablement un peu des trois. Toutefois, à la lueur des événements qui se sont déroulés par la suite, son constat revêt une tonalité prémonitoire :

"La vie juive a traversé un énorme bouleversement au cours des dernières cinquantesoixante années, et la perte de nos créations populaires est parmi les plus infortunées victimes de cette transformation. Avec la mort de chaque vieille personne, avec chaque incendie qui éclate, avec chaque exil que nous subissons, nous perdons un pan de notre passé. Le meilleur exemple de notre vie traditionnelle, nos coutumes et nos croyances disparaissent; les vieilles légendes poétiques et les chants et les mélodies seront bientôt oubliés; les anciennes et magnifiques synagogues tombent en ruine ou sont abandonnées aux flammes tandis que les plus précieux de nos ornements religieux se perdent ou sont vendus - souvent même à des non-juifs; les pierres tombales de nos pieux ancêtres sont englouties par la terre, leurs inscriptions sont effacées. Bref, notre passé, sanctifié par le sang et les larmes de tant d'innocents martyrs, s'évanouit et sera bientôt oublié » (An-Sky 1914: 10-11). 
En 1911, An-Sky ne possède toujours rien, nul foyer, épouse ou enfant ne l'ont fixé en un lieu. Il vit, ainsi qu'il l'a toujours fait, au jour le jour, ne sachant jamais de quoi le lendemain sera fait, les engagements de sa plume et de sa personne étant son seul gagne-pain. Sa liberté était donc absolue, mais elle dépendait cependant de sa force de persuasion pour mener à bien ses projets. Tout en envoyant ses chroniques quotidiennes sur le procès Beilis à vingt-huit journaux, An-Sky était parvenu, après une tournée de conférences dans les grandes villes russes auprès des organisations juives, à réunir des fonds suffisants pour lancer l'expédition. Il avait également persuadé le baron Vladimir Guenzburg, gendre du richissime Brodsky, d'en devenir le principal soutien financier. Au $1^{\text {er }}$ juillet 1912, elle démarre enfin à Ruzhin (aujourd'hui partie de Zhitomir en Ukraine), pour s'étendre dans les bourgades hassidiques de Rovno, Ostrog, Iziaslav, Miropol, Ostropol, Staro-Konstantinov, Polonoe, Slavuta et NovogradVolonski. Trois saisons de collecte dans les confins de l'Ukraine, de la Podolie et de la Volhynie, là où la modernité avait le moins accompli son œuvre, procurent des résultats inespérés. En 1913, il en témoigne dans une lettre à son mécène :

«Pendant ces derniers deux mois et demi, l'expédition a déjà sillonné 25 lieux (8 villescentres de district et 17 bourgades). Nous avons recueilli des matériaux dont le volume excède du double (ou même plus) celui recueilli par l'expédition de l'an passé; nous avons pris plus de 500 photos, nous avons enregistré presque 1000 airs musicaux et chansons, une énorme quantité de contes et légendes; nous nous sommes procuré 169 objets pour le musée, comprenant pas mal de choses anciennes et de grande valeur dont une vingtaine de manuscrits; la plupart de ces derniers sont des œuvres du $\mathrm{XVIII}^{e}$ et du XVII siècle qui n'ont jamais été publiées; il y a également beaucoup de lettres écrites par des personnalités connues, etc. Dans une des bourgades, j’ai réussi à exhumer d'un ancien cimetière (datant de l'époque de Chmielnicki) se trouvant dans une propriété privée [...], deux crânes, dont un semble avoir été fracturé par une arme. J'ai sorti ce crâne de la terre moi-même, d'une profondeur d'une archine [=0,71 m], je l'ai sorti de mes propres mains, et il ne fait pas doute que la fracture est ancienne. Dans ces lieux, j’ai pu noter beaucoup de légendes historiques très caractéristiques, remontant également au temps de Chmielnicki. Ce qui les caractérise est qu'elles sont répétées dans des lieux différents. Par exemple, dans 12 lieux, on m’a montré un endroit encerclé près de la synagogue où des fiancés ont été enterrés après avoir été tués par les haiddamaks pendant leur cérémonie nuptiale» (Lettre au baron Guenzburg, Archives de Kiev).

Ces matériaux inestimables n’ont pas encore fini de révéler leurs secrets, ni même d'être étudiés. Ils devaient être entreposés afin de fournir le fonds d'un musée ethnographique, mais les aléas de l'histoire en décidèrent autrement. Lorsqu'à l'été 1914 l'expédition doit interrompre l'enquête, la collection, destinée à rejoindre Saint-Pétersbourg, contient près de 700 objets, des manuscrits et pages de titres décorées, des objets rituels, 
1500 photographies d'anciennes synagogues avec leurs ornementations intérieures, des arches, des pierres tombales, sans compter les enregistrements musicaux, légendes et proverbes, comptines, et résultats des questionnaires. Une exposition parviendra à être organisée en 1917.

Entreprise sous les meilleurs auspices, l'expédition est inexorablement victime du démon de l'histoire. An-Sky avait auguré l'extinction de l'ancien monde juif, mais il n'avait certainement pas imaginé assister à son apocalypse. Transformée en ligne de front, la zone de résidence juive est parcourue par les troupes, livrée aux pogroms, ses habitants sont déportés. À la faveur de ses activités politiques, Rappaport peut accéder à tout l'appareil militaire russe, et il s'engage immédiatement dans le Comité de secours aux civils et victimes de guerre. Muni d'un saufconduit lui permettant de pénétrer dans l'enclave militaire, revêtu de l'uniforme, sabre au côté, il parcourt les bourgades, distribue aux survivants des médicaments, de la nourriture, du cognac; leur procure des infirmières et des nourrices, parvient à mettre sur pied des trains spéciaux, autorisés aux juifs. Il reprend simultanément sa plume de journaliste afin d'alerter l'opinion internationale, se rend secrètement à Saint-Pétersbourg pour sommer la Douma de prendre des dispositions de protection particulières pour les juifs de Galicie et de Bucovine, adresse une lettre ouverte au Saint-Synode. Puis, il retourne sur ses pas, retrouve les synagogues et les cimetières dévastés. Les cahiers de notes qu’il avait tenus lors de l'expédition se muent en journal de guerre. Son témoignage se veut précis : dates, lieux et déroulement des faits, compte rendu exact de ses activités. D'une localité à une autre, il prend le train - tant qu'ils roulent - et, s'il n'hésite pas à rapporter les propos odieux que tiennent ses compagnons de voyage sur les juifs, il ne s'appesantit guère sur la description des monstruosités vues ou racontées à chaque étape. Parfois cependant, une vision l'empoigne :

«La nuit était claire, une lune pleine s'élevait dans un ciel étoilé. Je me détournai pour rentrer lorsqu'une vision fantastique se révéla, devant nos yeux se dressaient des centaines de hautes pierres tombales, embrasées, rouges, gravées de lettres hébraïques incandescentes. Au premier instant, je n'ai pas réalisé ce qui se passait. Ce n'est qu'après que je compris qu'un cimetière juif était face à nous. Sous le feu des flammes, les stèles rougeoyaient. C'était une image hors du commun, fantastique. Exactement comme si des centaines de générations, des siècles de juifs, étaient sortis du passé pour regarder, avec des yeux brûlants, les atrocités qui se perpétraient dans leur shtetl en cette nuit limpide de pleine lune» (in 1920-1928: V, 102).

Engagé dans la guerre, Rappaport/An-Sky ne perd cependant pas de vue sa mission de collecte. À chaque permission, il rentre à Petrograd, lourdement chargé d'objets recueillis parmi les décombres, et note 
soigneusement les récits rapportés par les témoins des tragédies. Ce journal sera son dernier ouvrage, Khurbn Galitzye ("La Destruction de la Galicie», in ibid.: IV, V et VI), auquel il ne mettra le point final que dans les derniers instants de sa vie et qui ne paraîtra qu'après sa mort.

En dépit de l'importance inégalée du témoignage et de sa valeur historique, La Destruction de la Galicie n'est pas l'œuvre qui a fait entrer An-Sky dans le panthéon de la littérature yiddish du début du XXe siècle. Il n'a été traduit que très récemment en anglais et son audience n'a jamais, jusque-là, dépassé le cadre très limité du lectorat yiddishiste. Pourtant, inhumé avec les honneurs d'un homme d'État, sous le même mausolée que Y. L. Peretz et Y. Dinesohn à Varsovie le 6 novembre 1920, ses funérailles - grandioses selon les témoins - attestent son intronisation parmi les «grands». Par un sursaut narquois du démon, l'intégrale de l'œuvre d'An-Sky fut récupérée, lors de sa dernière décennie et, surtout, durant les deux dernières années qu'il passa en Pologne, par le patrimoine yiddish. Les générations qui suivirent ne surent pas que son œuvre avait, principalement, été composée en russe.

\section{La dernière valse de l'envahisseur}

"L'idée [de la pièce] m'en est venue lorsque je traversais les provinces de Podolie et de Volhynie pour en collecter le folklore. Arrivant, avec Engel à Iarmolinets, une bourgade de Podolie, nous ne trouvâmes pas d'auberge. L'homme riche du lieu nous hébergea [...] il avait une fille, enfant unique de 17 ou 18 ans, ravissante, solitaire, avec un long visage pâle et deux grands yeux profonds. Modeste comme la chasteté incarnée, elle parlait à peine [...]. Mais, quand le shabbes, un jeune étudiant de la yeshiva venait dîner chez eux, beau garçon, avec des yeux bleus rêveurs et une paire de longues papillotes bouclées, la jeune fille devenait alors une autre personne [...]. Ces deux jeunes âmes pures étaient attirées l'une par l'autre comme par magnétisme, à l'insu de tous et peut-être, qui sait, d'eux-mêmes? Le dernier shabbes, après la havdalah, assis avec un verre de thé, le riche hassid fut particulièrement disert. Il me parla de ses affaires et de son projet de marier sa fille au fils d'une riche et prestigieuse famille [...]. Il me vint à l'esprit qu'une tragédie allait se produire dans cette maison. À partir de ce soir-là, je me mis à imaginer les différentes voies qu'elle pouvait emprunter pour se perpétrer. Un soir, alors que, déjà rentré à Saint-Pétersbourg, je triais les récits et légendes de dybboukim que j'avais collectés, je m'arrêtai sur l'une d'entre elles. Dès le lendemain, je griffonnai dans mon carnet de notes les premiers vagues contours de je ne savais trop quoi [...]. Entre-temps la guerre mondiale avait éclaté [...], mettant un terme à mes visées littéraires. Je me suis engagé comme sauveteur et ne me suis plus préoccupé que du sort de mes frères de Galicie [...]. Je me trouvais là-bas dans un environnement mystique, qui le devint encore plus au cours des horribles persécutions et souffrances qui rappelaient l'époque des croisades et les massacres de 1648 . J'ai vu des ombres, des visages fermés, qui semblaient flotter dans les sphères de l'autre monde [...]. Une fois, je suis rentré dans la maison d'études de Gorliz après l'office du soir. Tu te rappelles la scène dans le premier acte de mon Dybbouk? C’est une réplique 
exacte de cet endroit, tel que je l'ai vu dans la lumière hivernale. Dès ce moment, le thème a commencé à prendre forme. J'ai écrit le premier acte à Tarnow. Le second une semaine plus tard, toujours en Galicie [...]. Le dernier, je l'ai écrit à Moscou " (in Zitron $1922: 85-86$ et AA. VV. $1980: 24-25$ ).

Lorsque l'exposition de la moisson ethnographique fut organisée à Petrograd en 1917, elle est accueillie par l'hospice juif installé dans l'île Vassilevski. Toujours sans permis, c'est là que dormait An-Sky durant l'un de ses passages clandestins en ville. Sur un grabat de l'hospice, il avait lu à un musicien de ses connaissances, Marc Rivesman, une ébauche inachevée du Dybbouk, tout en profitant de l'intérêt admiratif de son auditeur pour se faire nourrir. Le récit que celui-ci en a laissé dans ses mémoires est l'un des rares qui rendent la truculence et le côté bouffon du personnage, sans se soucier de lui conférer la solennité due à la mémoire d'un grand homme (cf. Beizer 1989: 94-95). La virulence de Rappaport se découvre également au détour de quelques lettres d'excuses envoyées, après d'inlassables discussions de comités divers, à l'un ou l'autre des protagonistes qu'il s'avisait d'avoir probablement froissé ou humilié durant la soirée. Mais sa correspondance, notamment avec le baron Guenzburg, laisse aussi entrevoir la vulnérabilité de l'homme d'entre les mondes. Ballotté par les événements, soumis au bon vouloir de ses commanditaires, l'incertitude et la précarité de son quotidien sont palpables. Juste avant d'entamer l'expédition ethnographique, il fait part de ses craintes :

"Je suis pétri d'incertitude. Comment tout cela va-t-il se passer? Pourrais-je gagner la confiance de ces pauvres gens ignorants? De ce monde dont je suis moi-même issu, mais dont je me suis tant éloigné pendant ces années? Il y a des moments où je me sens terriblement angoissé. Mais en même temps, de mon âme s'élève un sentiment immense de joie, procuré par le commencement de l'accomplissement du rêve de toute ma vie» (Correspondance, Archives de Kiev).

Dès octobre 1914, il dépêche une première mouture du Dybbouk (malheureusement perdue à ce jour) au baron, le priant instamment de réagir, comme celui-ci l'indique en réponse :

«[...] vous exigez une analyse de tous les aspects, du point de vue artistique ainsi que du point de vue psychologique et théâtral. Pour dire cela autrement, je devrais faire ce qui correspond à la vocation d'un critique littéraire de théâtre. Mais moi, je ne possède ni l'expérience, ni les connaissances nécessaires, ni, il faut le souligner, l'impartialité requise. Je suis trop incliné à trouver votre travail parfait; je suis trop envoûté par le tableau que vous avez créé» (Lettre du baron Guenzburg, Archives de Kiev).

La pérennité de cette pièce se révèle tout aussi mystérieuse que son succès. Car les premières séances de lecture publique, organisées à Moscou, Varsovie, Vilno ou Kiev entre 1917 et 1918, sont loin de recevoir 
un accueil favorable. L'un des auditeurs se souvient qu'à Varsovie, l'auditoire réticent n'a pas même osé faire part de ses impressions à un An-Sky fragile et diminué par la maladie. À Kiev, il en est allé autrement. La scène se déroula lors d'une conférence organisée par la communauté. Le grand poète Nachman Bialik, ami d'An-Sky depuis son retour à Saint-Pétersbourg en 1909, était venu l'écouter et, prié de réagir, peut-être un peu pris de court, lâcha brutalement:

«[...] j’ai l'impression, que toi, en tant que collecteur de folklore, tu t'es penché sur toutes les poubelles et que tu y as choisi des détritus que tu as assemblés pour en faire une histoire, comme un tailleur rajuste des hardes pour en faire une nouvelle robe. Tu as passé toutes tes années chez les goyim, à la fin de tes jours, à présent que tu es à moitié mort, tu es venu chez nous, les juifs. Et le décret céleste est tombé sur toi afin que tu ramasses le folklore dans les poubelles, et c'est de cet assemblage qu'est issu ton Dybbouk» (in Ungerfeld 1964 : 195).

On peut aisément imaginer l'atteinte portée à celui qui rêvait de rendre la profondeur de l'âme juive face à une réaction à la fois si cruelle pour l'œuvre et si odieuse pour son auteur.

Mais le démon avait encore un tour dans son sac. Pour se racheter, Bialik promit de traduire la pièce en hébreu. Entre-temps, la révolution d'Octobre avait éclaté, et Rappaport, renouant avec ses rêves, élu député à la Douma au titre du Parti SR qui avait remporté les premières élections libres, reprend du service. Guère pour longtemps. L'insurrection bolchevique met les membres du parti hors-la-loi, leurs vies sont en danger. Rappaport doit reprendre le bâton de l'exil.

Déguisé en pope, chargé de deux lourdes valises pleines de ses matériaux ethnographiques les plus précieux, Semion Akimovitch Rappaport quitte la Russie en septembre 1918. C'est en homme brisé et malade qu'An-Sky parvient à Vilno sous occupation allemande. Il y ouvre un musée ethnographique juif, établit la Ligue culturelle. En 1919, lorsque Ayzik Meyer Devenishski (Weiter), l'un de ses amis proches, est massacré au cours du pogrom, son cœur lui fait défaut : sévèrement affaibli, il se traîne vers Varsovie. Ses dernières lettres attestent la lassitude :

"J'en ai tant surmonté, deux années d'errance en Galicie suivies de la Première révolution!; les activités politiques en tant que membre de la Douma à Petrograd; puis l'Assemblée constituante au Conseil d'État provisoire, ensuite la Seconde révolution dans les rangs des "contre-révolutionnaires"; septembre 1918, la fuite de Russie, tous les matériaux ethnographiques emballés et laissés à la communauté de Moscou ; le musée ethnographique abandonné à l'arbitraire. Qui sait s'il en reste encore le moindre vestige? Parti pour Vilna, j'ai survécu à ce qui s'y est passé, mais mon cœur en a subi les conséquences, malade, j'ai cru être parvenu à ma fin. J'ai dû renoncer à toute activité politique et communautaire» (in Rechtman 1958: 22). 
L'épilogue est imminent, mais il joue encore avec un pâle espoir :

«En ce qui me concerne aucune nouvelle réjouissante à écrire. Je suis encore malade. Mes jambes sont enflées et je peux à peine marcher. Je compte d'ici quelques mois... peut-être... pouvoir aller à Berlin" (in Ibid.).

Il s'éteint un mois après sa dernière lettre, le 6 novembre 1920.

Au cours de la fuite de Rappaport/An-Sky, l'original du Dybbouk s'égara. Était-il en russe, comme le laisse présager l'envoi du manuscrit au baron Guenzburg? L'avait-il ensuite traduit en yiddish pour des auditoires communautaires? À moins que les archives russes n'en exhument la trace, nous n'en saurons rien. Toujours est-il que le texte qui entra dans le panthéon de la littérature yiddish fut celui rédigé en hébreu par le poète Nachman Bialik, et de manière cocasse. Il s'en expliqua lors d'une réunion de la troupe de théâtre juif Habimah, à Jérusalem, au printemps 1923:

«[...] je vais vous dire la vérité. En fait, ce n'est pas avec grand désir ni en y croyant particulièrement que je me suis retrouvé dans cette affaire. Cette pièce ne m'avait pas particulièrement plu, et je ne m'en étais pas caché auprès de son auteur. Il avait lu sa pièce devant une salle, à Kiev. J'y étais et il m’a prié de communiquer mes réactions et c'est ce que j'ai alors fait publiquement" (in Ungerfeld 1964 : 195).

Bialik n'avait guère envie de tenir sa promesse de traduire le texte, mais, harcelé par l'éditeur de la revue Ha-tekufah qui tenait à lancer son premier numéro avec sa signature, il en fit une traduction dont il était si peu fier, qu'il tenta de ne pas faire apparaître son nom (cf. An-Sky 1918).

Sans rancune, An-Sky le remercia dans une lettre où il lui dit avoir pleuré d'émotion à la lecture du texte. Et la reprise de la pièce en yiddish, intitulée alors Tsvishn tsvey veltn ("Entre deux mondes»), se fit à partir du texte hébraïque. Devenue la carte de visite de la troupe Habimah, l'œuvre dans ses deux versions, hébraïque et yiddish, reçut un accueil si spectaculaire que Shloyme Zanvl Rappaport pénétra sous le masque d'An-Sky parmi les immortels et que le Dybbouk, après avoir intégré le répertoire des pièces de théâtre, demeure un classique du cinéma expressionniste parlant yiddish, réalisé par Michael Waszyński à Varsovie en 1937. 
La biographie que j'aurais pu écrire aurait conjugué tous les temps. S’y seraient mêlés les genres et les personnages, tous masqués sous des noms d'emprunt. Le dybbouk aurait mené la danse, niché dans la barbe de Lavrov, sous la robe du Père Gapone, il aurait dansé dans les allées du cimetière de Varsovie ; il se serait glissé sous la table d'une société savante de Vilno, aurait enflammé le discours d'un professeur de Leningrad, éparpillé les boîtes empilées d'une archiviste de Kiev, semé le désordre dans une exposition à New York, mélangé en chantant les Archives du peuple juif à Jérusalem, puis, s'échappant d'un sac à dos, il se serait installé dans le tiroir de mon bureau. Le démon aurait échappé à l'histoire pour mieux s'y dissimuler sous couvert de légendes, de croyances et de coutumes exigeant leur dû. La biographie aurait embarqué par le train des exilés russes, traversé leurs colonies de Berlin, Paris, Londres, Genève, Berne, Zurich ou Königsberg, elle y aurait toujours rencontré le même homme, déguisé chaque fois différemment, parlant d'une autre voix. Le dybbouk aurait alors exhumé des mémoires de papier un monde glacé, l'aurait réchauffé avec de la vodka en lui racontant qu'il hante toujours le présent, enfin, la biographie aurait soufflé sur ces morts pour qu'ils vivent.

École des hautes études en sciences sociales Centre de recherches historiques, Paris sag@ehess.fr

MOTS CLÉS/KEYWORDS: Semyon A. An-Sky - Le Dybbouk, 1918 - biographie/biography yiddish - exil/exile - culture juive/Jewish culture - persécution des juifs/persecution of Jews Parti socialiste-révolutionnaire (Russie)/Socialist-Revolutionary Party (Russia) - Révolution de 1905/1905 Russian revolution - guerre civile russe/Russian civil war - Révolution bolchevique/Bolshevik revolution - Empire russe/Russian empire. 


\section{- AN-SKY, Shloyme RAPPAPORT, Semyon Akimovitch}

1894 Ocherki Narodnoi Literatury. St-Pétersbourg. [Trad. : Narod i Kniga (= Le Peuple et le Livre), Moscou, 1914.] 1907 Chto Takoe Anarkhizm? (= Qu'est-ce que l'anarchisme?), in Sobranie Sochinenii... St-Pétersbourg, 1909.

1908 Evreiskoie Narodnoe Tvorchestevo (= L'Art populaire juif ou L'Ethnopoétique juive), in Perezhitoe. [Trad. : "Di Yiddishe Folks-Shafung ", in Gezamelte Schriftn... Warsaw-New York, 1920-1928, t. XV.] 1909 Sobranie Sochinenii (= Euvres complètes). St-Pétersbourg, 2 vol.

1911 Gezamelte Schriftn (= Euvres complètes). New York-St-Pétersbourg, 2 vol. 1914 Dos Yidishe Etnografishe Program (= Le Programme ethnographique), 1 : Der Mensh. Ed. by L. I. Sternberg. Petrograd, Publication of the Jewish Etnographic Expedition. 1918 «Ben Shnei Olamot (= Entre deux mondes) ", trad. par Chaim Nachman Bialik, Ha-tekufah 1 : 222-296.
1920-1928 Gezamelte Schriftn in Funfsn Bander, mit der Biografye un Bilder fun Oyter Sh. An-Ski Shloyme Zaynvl Rappaport (= Euvres complètes, avec biographie et photographies de l'auteur).

Warsaw-New York, 15 vol.

1964 Oysgeklibner Shriftn. Muzik tsu Lider (= Morceaux choisis. Poésies, proses, théâtres, essais). Buenos Ayres, Yoysef Lifshits-Fond fun der Literatur-Gezelshaft baym Yivo.

1992 The Dybbuk and Other Writings. Ed. and with an introd. by David G. Roskies. New York, Schocken Book.

2003 Khurbn Galitzye (= The Enemy at his Pleasure. A Journey Through the Jewish Pale of Settlement During World War I). Transl. by Joachim Neugroschel. New York, Metropolitan books-Henry Holt \& Co.

Fonds d'archives An-Sky. Bibliothèque Vernadsky, bibliothèque nationale de Kiev, manuscrits orientaux, section judaica, f. 339 .

\section{- Catalogues d’expositions \& livraisons spéCIales}

AA. VV.

\section{Yiddisher Folks-Ornoment,} Tsuzamngeshtelt fun Sh. Yeduvin un M. Malkin (= Ornements populaires juifs, rassemblés par Sh. Yedoubin et M. Malkin). Vitebsk, Perets-gezelshaft. [Rééd. : Tel-Aviv, 1970.]

1980 S. Ansky (1863-1920). His Life and Works, November 1980. New York, Yivo Institute for Jewish Research.

1994 Back to the Shtetl. An-Sky and the Jewish Ethnographic Expedition, 1912-1914 [en hébreu]. Jerusalem, Catalogue of the Israeli Museum.
Beukers, Mariëlla \& Renée Waale, eds 1992 Tracing Ansky. Jewish Collections from the State Ethnographic Museum in St Petersburg. Zwolle, Waanders Uitgevers / Amsterdam, Joods Historisch Museum.

Les Cahiers du judaïsme

2005-2006 Les Cahiers du judaïme 19: An-Sky, un homme entre les mondes.

\section{Di Goldene Keyt}

1964 Di Goldene Keyt 48 : Tsum Hundertsn Geboyrn Tog fun Sh. An-Sky (S. Rappaport) (= Pour le centenaire de la naissance d'An-Sky [S. Rappaport]). 
Rakitin, Vasilii \& Andrei Sarabianov, eds

1994 An-Sky Semyon, The Jewish Artistic

Heritage. An Album. Moscow, "RA".

Rechtman, Abraham

1958 Yiddishe Etnografye un Folkor

(= Folklore et ethnographie juives).

Buenos-Ayres, Yiddisher Vissentshaftlekher Institut.

Safran, Gabriella \& Steven J. Zipperstein, eds 2006 The Worlds of S. An-sky. A Russian Jewish Intellectual at the Turn of the Century. Stanford, Stanford University Press.
Shalit, Moyshé, ed.

1920-1921 Lebn. Heftn fun Tseyt tsu Tseyt far Literatur, Kunst un Publitsistik 8-9.

1937 Fun Noentn Over, Dershaynt mit der Mithilf fun der Vilner Historisher Etnografisher Gezelshaft Oyfn Nomen fun S. An-Ski (= Du passé proche, publié avec le soutien de la Société ethnographique de Vilno au nom de S. An-Sky), 1. Warsaw.

\section{- Autres RÉFÉRENCES}

\section{Beizer, Mikhail}

1989 The Jews of St Petersburg.

Excursions Through a Noble Past.

Philadelphia, The Jewish Publication

Society.

\section{Biale, David}

2002 "A Journey Between Worlds", in David Biale, ed., Cultures of the Jews. A New History. New York, Schocken Books : 799-860.

2005 «Un voyage entre les mondes : la culture juive d'Europe de l'Est des partages de la Pologne à l'Holocauste", in David Biale, ed., Les Cultures des juifs. Une nouvelle histoire. Paris-Tel-Aviv, Éd. de l'éclat.

\section{Chernov, Victor}

1948 Yiddish Tuer in der Partey

Sotsialistn Revolutsionern (= Militants juifs du Parti social-révolutionnaire).

New York, Shoulson Press.

Doubnov, Simon

2001 Le Livre de ma vie.

Souvenirs et réflexions, matériaux

pour l'histoire de mon temps.

Paris, Le Cerf.
Frankel, Jonathan

1981 Prophecy and Politics. Socialism, Nationalism, and the Russian Jews (1862-1917). Cambridge-New York, Cambridge University Press.

Graubart, Pinhes

1928 An Ander Lebn (= Une autre vie).

Warsaw, Kultur-Liga.

Klier, John D. \& Shlomo Lambroza, eds 1992 Pogroms. Anti-Jewish Violence in Modern Russian History. CambridgeNew York, Cambridge University Press.

Krutikov, Michael

1994 «Brivn fun Sh. An-Ski tsu Khaim Zhitlowski (= Lettres d'An-Sky à Chaim Zhitlovsky)", Yivo bletter 2 : 281-312.

Nathans, Benjamin

2002 Beyond the Pale. The Jewish Encounter with Late Imperial Russia. Berkeley-

Los Angeles, University of California Press.

Orlov, Alina

2005 «From Zero to Ten : Discovering Yudovin's Photographs in Post-Soviet Russia ", East European Jewish Affairs 35 (1) : 3-6. 
Reysen, Zalmen

1928 "An-Sky", in Leksikon fun den

Yiddisher Literatur Presse un Filology

(= Lexique de la littérature, de la presse et

de la philologie en yiddish). Vilna: I, 125-141.

\section{Roskies, David}

1984 Against the Apocalypse. Responses to Catastrophe in Modern Jewish Culture.

Cambridge, Harvard University Press.

1990 "The Maskil as Folk Hero",

Prooftexts 10 (2) : 219-235.

1992 «S. Ansky and the Paradigm of

Return ", in Jack Wertheimer, ed., The Uses

of Tradition. Jewish Continuity in the Modern

Era. New York-Jerusalem, Jewish

Theological Seminary of America : 243-260.

Rozhanski, Shmuel

1964 «Sh. An-Ski, fun a Narodnik a

Meshulekh : A Zeltn Figur far a Historisher

Roman (= Sh. An-Sky, du Narodnik

à l'Émissaire: figure pour un roman

historique) ", in Semyon A. An-Sky,

Oysgeklibner Shriftn, Muzik tsu lider

(= Morceaux choisis, Poésies, proses,

théâtres, essais)... op. cit. : 13-24.

\section{Shalit, Moyshé}

1929 "Der An-Ski Arkhiv, Zayn Inhalt un

Vert (= Les archives An-Sky, leur contenu et importance) ", Literarishe Bletter 259:

313-314.

Trunk, Isaiah

1982 "Homer Bilti Yadua Shel Mishlahat

An-Ski Ba-Shanim 1912-1916

(= Matériaux inédits de l'expédition

An-Sky des années 1912-1916)",

Gal Ed $6: 229-245$.

\section{Ungerfeld, Moshé}

1964 «Foler Brivvoystoysh Tsvitshn Sh. Anski un Kh. N. Bialik (= Feuillets de la correspondance entre Sh. An-Sky et Ch. N. Bialik) ", Di Goldene Keyt 48 : 194-210.

Weinberg, David H.

1996 Between Tradition and Modernity.

Haim Zhitlowski, Simon Dubnow, Ahad $H a-A m$, and the Shaping of Modern Jewish Identity. New York, Holmes \& Meyer.

Werses, Shmuel

1986 " "Between Two Worlds"

(The Dybbuk): A Textual History", in Studies in Yiddish Literature and Folklore. Jerusalem, Hebrew University of Jerusalem.

Zeitlin, Hillel

1921 «Der Lebnsveg fun Sh. An-Sky

(= L'itinéraire de Sh. An-Sky) ", in

Almanakh tsum 10-Yerikn Yubileum

fun «Moment». Warsaw : 49-73.

Zerubavel, Yaakov

1964 "Sh. An-Sky, Shtrikhn tsu Zayn Kharakteristik (= De quelques aspects caractéristiques de Sh. An-Sky)", Di Goldene Keyt 48 : 5-15.

Zhitlovski, Chaim

1935 Zikhrones fun meyn Lebn (= Mémoires de ma vie). New York, Shoulson Press.

\section{Zitron, Shmuel Leyb}

1922 Drey Literarishe Doyres (= Trois générations littéraires). Vilna, S. Srebernik, 4 vol. 
Sylvie Anne Goldberg, Une valse du démon et de l'histoire. - Le Dybbouk de Shloyme An-Sky est l'une des œuvres les plus connues de la littérature yiddish du début du XXe siècle. Depuis l'ouverture des archives de l'ex-URSS, son auteur a été, à son tour, exhumé de l'oubli et les innombrables travaux qui lui ont été consacré dans la dernière décennie l'ont transformé en figure paradigmatique du monde juif russe de la période révolutionnaire. Mais un paradigme ne s'obtient qu'en forçant les traits, ou en réduisant les couleurs qui «font» une personne. La tentative biographique menée ici tente de dénouer quelques pièges posés entre la fixité des sources existantes et les constructions opérées par les témoignages littéraires. Au-delà de l'enjeu historiographique, cet essai voudrait également dépasser les cadres de la mémoire objective afin de montrer comment les enjeux du passé peuvent, parfois, se rejouer dans le présent.
Sylvie Anne Goldberg, A Walz with the Devil and History. - Shloyme Ansky's "The Yiddish literature of the early $20^{\text {th }}$ century Since the opening of the archives of the former UsSR, its author has been exhumed from oblivion, and the countless studies which have been devoted to him in the last decade have transformed him into a paradigmatic figure of the Jewish Russian world of the revolutionary period. However, when creating a paradigm, one inevitably distorts or diminishes the distinctive traits that "make" a full person. The biographical sketch attempted here seeks to avoid some traps set between the fixity of available sources and the constructions of literary evidence. Beyond the historiographic stakes, this essay also aims as well to go beyond the framework of objective memory, in order to show how the contests of the past may, sometimes, be replayed in the present. 